\title{
Impact of controlling atrial fibrillation on outcomes relevant to the patient: focus on dronedarone
}

This article was published in the following Dove Press journal:

Patient Related Outcome Measures

17 December 2012

Number of times this article has been viewed

\section{Anwar A Chahal \\ Omer Ali \\ Ross J Hunter \\ Richard J Schilling}

Department of Cardiology Research, St Bartholomew's Hospital, London, United Kingdom
Correspondence: Richard J Schilling Department of Cardiology Research, First Floor, Dominion House, 60 Bartholomew Close St Bartholomew's Hospital, West Smithfield, London, ECIA 7BE, United Kingdom

Tel +44203465 5398

Fax +44203465 577।

Email r.schilling@qmul.ac.uk
Abstract: Atrial fibrillation (AF) is a substantial cause of mortality and morbidity in the Western world. It is a massive burden on health care systems, and its prevalence is expected to double over the next 20 years. Trials evaluating antiarrhythmic drugs or catheter ablation have focused on recurrence of arrhythmia, perhaps neglecting outcomes relevant to patients, such as symptoms, need for antiarrhythmic drugs, need for hospitalization, and rates of stroke and death. An association has been demonstrated between sinus rhythm and survival in several studies, and there is evidence emerging that successful catheter ablation may reduce rates of stroke and death. Similarly, dronedarone has been shown to reduce hospitalizations and death in patients with paroxysmal AF or persistent AF of recent onset, although it may cause adverse events in permanent AF. New antiarrhythmic drugs are a welcome addition to the armamentarium, since there are limitations to current antiarrhythmic drugs. In particular, sotalol, flecainide, and propafenone cannot be used safely in those with structural heart disease, and amiodarone has important adverse reactions that limit long-term use. Indeed, the use of conventional antiarrhythmic drugs may negate any survival benefit derived from maintaining sinus rhythm. Although dronedarone appears promising with respect to hard endpoints such as stroke and death in certain patients, it may not be safe for those with heart failure or those with permanent AF. Furthermore, the trials suggesting that dronedarone may impact on these endpoints were compared with placebo rather than with an active comparator group. Further "head-to-head" comparisons between dronedarone and other antiarrhythmic drugs are needed to determine whether this property is unique to dronedarone alone.

Keywords: atrial fibrillation, rhythm, antiarrhythmics, ablation, stroke, dronedarone

\section{Introduction}

Atrial fibrillation (AF) is the commonest cardiac arrhythmia, with a prevalence of $1 \%$ in the general population. ${ }^{1}$ This equates to 2.3 million US adults currently affected, with the number expected to increase to 7.5 million by 2050 , due in part to an ageing population. ${ }^{2}$ AF is associated with substantial morbidity and mortality from stroke, thromboembolism, and heart failure, and is an independent predictor of increased mortality. ${ }^{3,4}$ The economic burden is enormous, with an estimated annual cost of US\$ 26 billion for treating AF and its complications in the US alone. ${ }^{5}$

The association between AF and stroke is well recognized, and hence the priority in treating AF is to identify those at high risk of stroke and anticoagulate accordingly. There remains controversy regarding rate control versus rhythm control, but it is now accepted that rhythm control should be adopted for those who are symptomatic despite adequate rate control. However, determining which patients are symptomatic 
can be difficult because symptoms are often insidious and vague (palpitations, lethargy, shortness of breath, decreased exercise tolerance) and often attributed to ageing. Many patients tolerate and adapt lifestyles to their symptoms, not realizing the full impact of AF until sinus rhythm is restored. In this group, a therapeutic trial of cardioversion may be undertaken to see if symptoms are improved by maintaining sinus rhythm.

Randomized controlled trial data from the AFFIRM (Atrial Fibrillation Follow-up Investigation of Rhythm Management) study suggested no morbidity or mortality benefit of rhythm control over rate control with a strategy involving antiarrhythmic drugs and/or direct current cardioversion. ${ }^{6,7}$ However, subsequent reanalysis did show a significant survival advantage in those maintaining sinus rhythm, but it is unclear whether this relationship is causal or whether sinus rhythm is a marker for some other factors. ${ }^{8}$ Catheter ablation is significantly more effective in maintaining sinus rhythm than antiarrhythmic drugs. ${ }^{9-14}$ There is also emerging data suggesting that there may be some mortality benefit of curative catheter ablation, though this requires further prospective evaluation. ${ }^{15-18}$ Recent multicenter registry data also suggests that risk of stroke may be reduced after successful catheter ablation of AF, although the risk-benefit ratio of oral anticoagulation in this setting is still being prospectively evaluated through randomized trials. ${ }^{16,19}$ At present, catheter ablation is reserved for patients who have symptoms refractory to at least one antiarrhythmic drug. The evolution of percutaneous ablation techniques is causing a shift in management trends, with early ablation strategies aiming to halt progression to long-standing persistent or permanent AF. ${ }^{17,20}$

The current medical benchmark of success is arbitrarily set high (recurrence of AF or atrial tachycardia for $\geq 30$ seconds \pm symptoms). ${ }^{21}$ Although benchmarks are necessary to compare treatments, they do not necessarily correlate with measures of success from the patient's perspective. For example, those with recurrent arrhythmia after catheter ablation meeting this criterion often have good improvement in their symptoms. ${ }^{22}$ For patients, the most important outcomes are symptom improvement, avoiding medication, reduction of stroke risk and death.

At present, antiarrhythmic drugs remain first-line agents in managing AF. Of the agents used, amiodarone is the most closely studied and is also the most effective in maintaining sinus rhythm. ${ }^{23}$ Its use is usually restricted to the short-term due to the high incidence of major side effects, and hence there has been a tremendous drive to find a pharmacological alternative. Dronedarone is a novel structural analog of amiodarone which is recommended for rhythm control in AF in both European and US guidelines. ${ }^{20,24}$ In this review, we discuss the evidence for dronedarone and its potential role in treating $\mathrm{AF}$.

\section{Methods}

A systematic search of peer-reviewed literature using PubMed, MEDLINE, and the Cochrane Library databases was performed. The main body of text of the manuscript needed to be in English to minimize misinterpretation, and case reports were excluded. The search was performed separately by two independent researchers (OA and CAAC).

The query consisted of "atrial fibrillation", "antiarrhythmic drugs", "anti-dysrhythmic drugs", "catheter ablation", and "dronedarone". Abstracts of the identified articles were read on the basis of this information and eligible articles identified. Subsequently, full-text papers were downloaded or ordered. If a report could not be ordered through local library services, attempts were made to contact the authors. The available reports were read independently by two researchers (OA and CAAC) and classified according to the defined criteria. Efforts were made to filter duplicates; publications from the same institution were checked for the period of data collection. The format of the review was structured and analyses were performed according to the Cochrane guidelines. ${ }^{25}$

\section{Dronedarone: pharmacology and electrophysiological properties}

Dronedarone is a structural analog of amiodarone, but with two important differences, ie, lack of iodine on the benzene ring and a methane sulfonyl group added to the benzofuran ring. The removal of iodine is thought to be responsible for the better side effect profile, with a lower incidence of pulmonary fibrosis, skin photosensitivity, and ocular affects. The other change is thought to decrease lipophilicity, and thereby the markedly shorter half-life (20-40 hours versus 21-47 days) and reduced tissue accumulation. ${ }^{26,27}$

Like amiodarone, dronedarone is a multichannel blocker that inhibits sodium, potassium, and calcium channels. Hence it possesses in vitro electrophysiologic characteristics of all four Singh-Vaughan-Williams classes of antiarrhythmic action. ${ }^{28}$ Other effects include negative chronotropy, vasodilatory, antiadrenergic, and blood pressure-lowering properties. ${ }^{29,30}$

Dronedarone is highly absorbed after oral administration in healthy subjects. ${ }^{26}$ However, it undergoes significant firstpass metabolism, resulting in low systemic bioavailability, with an absolute bioavailability of $15 \%$. Coadministration 
with food increases bioavailability by 2 -fold to 4 -fold. Dronedarone is highly bound to plasma protein, ensuring it is quickly distributed throughout the circulation with a maximum plasma concentration attained within 3-5 hours.

Dronedarone is extensively metabolized, and mainly by the hepatic cytochrome P450 (CYP) system (CYP3A4). Like all drugs metabolized via this route, inhibitors of CYP can lead to reduced metabolism and increased serum concentration of dronedarone. The predominant circulating metabolite is formed by N-debutylation, followed by oxidation and oxidative deamination. The N-debutyl metabolite may contribute to the pharmacologic activity of dronedarone, but it is 3-10 times less potent. The metabolites are excreted primarily in feces $(>80 \%) .{ }^{31}$ The long half-life of amiodarone is a result of low clearance and a large volume of distribution. Dronedarone has a much smaller volume of distribution (mean 1440-3440 L), with steady state reached after $4-8$ days. $^{26}$

\section{Clinical trial data Effective dose}

DAFNE (Dronedarone Atrial FibrillatioN study after Electrical Cardioversion) was a double-blind, randomized, controlled trial which sought to determine the optimum dose required for maintaining sinus rhythm. ${ }^{32}$ Patients with persistent AF ( $\mathrm{n}=270, \mathrm{AF}>72$ hours and $<12$ months) were randomly allocated to 800,1200 , or $1600 \mathrm{mg}$ daily doses of dronedarone or placebo. Spontaneous cardioversion occurred in only $5.8 \%-14.8 \%$ of patients on dronedarone (with a dosedependent effect) after 5 days $(P=0.026)$, with the majority undergoing electrical cardioversion. The primary endpoint of time to AF relapse increased on dronedarone $800 \mathrm{mg}$, with a median of 60 days versus 5.3 days in the placebo group (relative risk reduction of recurrence 55\% [95\% confidence interval [CI] 28-72, $P=0.001)$ with no proarrhythmia noted. At higher doses, patients reported gastrointestinal upset, and at $1600 \mathrm{mg}$ QT prolongation was noted (although with no proarrhythmia). There were no thyroid abnormalities in any of the groups.

The subsequent Phase III EURIDIS (EURopean trial In atrial fibrillation patients receiving Dronedarone for the maintenance of Sinus rhythm) and ADONIS (Americanaustralian-african trial with DronedarONe In atrial fibrillation or flutter patients for the maintenance of Sinus rhythm) trials used dronedarone $400 \mathrm{mg}$ dosing twice daily. In EURIDIS, the median time to AF recurrence was 41 days in the placebo group versus 96 days in the dronedarone group. ${ }^{33}$ In ADONIS, the median time to AF recurrence was 59 days on placebo and 158 days on dronedarone. ${ }^{33}$ In these studies, recurrence of AF was accepted as an episode lasting more than 10 minutes, in contrast with 30 seconds for catheter ablation studies.

It is also important to note the selection criteria for both these trials included patients of either gender, age over 21 years, at least one episode of AF in the preceding 3 months, and being in sinus rhythm before randomization for at least one hour. Those deemed to have permanent AF were excluded. Patients previously treated with amiodarone were also enrolled once amiodarone was discontinued.

The post hoc analysis of EURIDIS and ADONIS showed a $27 \%$ relative risk reduction in hospitalization for cardiovascular causes and death for patients treated on dronedarone versus placebo. ${ }^{33}$ However, standard therapy was allowed in both arms, including rate control agents (beta-blockers, calcium antagonists, and/or digoxin).

\section{Effects on hospitalization}

This beneficial effect on survival was demonstrated again in ATHENA (A placebo-controlled, double-blind, parallel arm Trial to assess the efficacy of dronedarone $400 \mathrm{mg}$ bid for the prevention of cardiovascular Hospitalization or death from any cause in patiENts with Atrial fibrillation/atrial flutter). ${ }^{30}$ ATHENA is the largest $(n=4628)$ single antiarrhythmic drug trial conducted in AF. This trial used cardiovascular hospitalization or death as outcome measures in contrast to recurrence of AF by electrocardiographic criteria. ${ }^{34}$ The trial demonstrated that dronedarone reduced a composite of cardiovascular hospitalizations or all-cause death by $24 \%$. For hospitalization because of AF alone, there was a $37 \%$ reduction in the dronedarone arm compared with placebo. This benefit was consistent across all subgroups, including those patients who remained in AF. The ventricular rate response during AF was reduced by $10-15$ beats per minute on those taking dronedarone. However, ATHENA also compared dronedarone with placebo rather than an active comparator group. Therefore, it is perhaps not surprising that patients were better rate-controlled and presented to hospital less often requiring treatment. This has led some to conclude simply that medical treatment of AF reduces this composite endpoint.

This effect on ventricular rate was confirmed in ERATO (Dronedarone for the control of ventricular rate in permanent atrial fibrillation: The Efficacy and safety of dRonedArone for The cOntrol of ventricular rate during atrial fibrillation) study). ${ }^{35}$ Furthermore, ERATO demonstrated sustained incremental rate control at rest and during exercise by dronedarone in patients with permanent AF treated with standard rate-controlling drugs. The safety profile in ERATO was good, with no evidence of proarrhythmia. 


\section{Use in NYHA classification III/IV or recently decompensated heart failure}

The ANDROMEDA (ANtiarrhythmic trial with DROnedarone in Moderate to severe CHF Evaluating morbidity DecreAse) trial specifically enrolled patients with New York Heart Association (NYHA) functional class III or IV heart failure and recently decompensated heart failure. ${ }^{36}$ This trial ran at a similar time to ATHENA. In contrast with earlier trials demonstrating a mortality benefit and a good safety profile, ANDROMEDA showed worse mortality in the dronedaronetreated arm (13.8\% versus 8\%; hazard ratio 2.13 ). This trial was not designed to study patients with AF, but a mortality benefit of dronedarone in patients with heart failure (only $25 \%$ of patients had AF at randomization). The trial was stopped prematurely after an interim safety analysis revealed an excess of deaths in the dronedarone-treated arm. The risk of death was greatest in patients with severely impaired left ventricular function. The adverse outcomes were thought to be due in part to more frequent discontinuation of angiotensin receptor blockers or angiotensin-converting enzyme inhibitors, which were stopped due to a rise in creatinine levels, interpreted as worsening renal function. These rises in creatinine are in fact thought to be due to the inhibitory effects of renal tubular excretion of creatinine, which are well established and known not to affect glomerular filtration rate. ${ }^{37}$ Although the aim of ANDROMEDA was to determine favorable outcomes in patients on dronedarone compared with placebo for those with moderate to severe cardiac failure, the low number of patients with AF (25\%) may have contributed to the lack of efficacy, because the antiarrhythmic properties of dronedarone may not offer any benefit to patients without AF.

\section{Use in permanent AF}

PALLAS (the Permanent Atrial fibriLLAtion Study using dronedarone on top of standard therapy) was a randomized, double-blind, placebo-controlled, parallel-group trial which assessed the clinical benefit of dronedarone $400 \mathrm{mg}$ twice daily on top of standard therapy in patients with permanent $\mathrm{AF}$ and additional risk factors, and was a large outcome trial intended to evaluate the effectiveness of dronedarone in reducing major vascular events or death in patients with permanent AF. ${ }^{38}$ The first coprimary outcome was stroke, myocardial infarction, systemic embolism, or death from cardiovascular causes. The second coprimary outcome was unplanned hospitalization for a cardiovascular cause or death. The trial was stopped early due to safety reasons during an interim data analysis. The dronedarone-treated arm had increased rates of heart failure, stroke, and death (21 in the dronedarone arm versus 10 in the placebo).

\section{Amiodarone versus dronedarone}

In DIONYSOS [randomized double blind trial to evaluate the efficacy and safety of dronedarone (400 mg BID) versus amiodarone (600 mg daily for 28 days, then $200 \mathrm{mg}$ daily thereafter) for at least 6 months for the maintenance of sinus

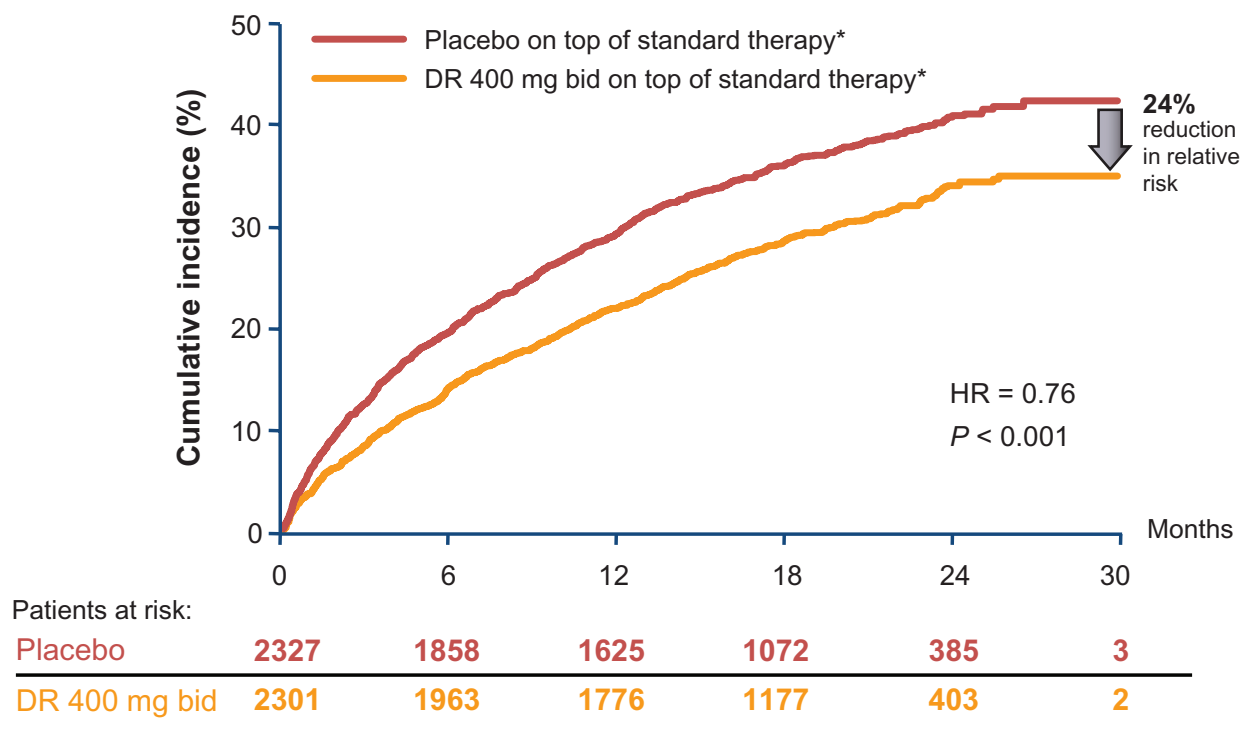

Figure I Impact of dronedarone on the composite endpoint of cardiovascular hospitalizations or death in the ATHENA trial.

Notes: *Standard therapy may have included rate control agents (beta-blockers, and/or Ca-antagonist and/or digoxin) and/or anti-thrombotic therapy (Vit. K antagonists and lor aspirin and other antiplatelets therapy) and/or other cardiovascular agents such as ACEls/ARBs and statins.

Reproduced with permission from The New England Journal of Medicine Adopted from Hohnloser SH et al. ${ }^{30}$ 
rhythm in patients with atrial fibrillation $(\mathrm{AF})], 504$ patients were randomized to dronedarone $(n=249)$ or amiodarone $(n=255)$ for a minimum of 6 months. ${ }^{39}$ The primary composite endpoint (recurrence of AF, including unsuccessful direct current cardioversion or premature study discontinuation) was $75.1 \%$ (dronedarone) and 58.8\% (amiodarone) at 12 months (hazard ratio 1.59; 95\% CI 1.28-1.98; $P<0.0001)$. This was largely driven by AF recurrence with dronedarone compared with amiodarone $(63.5 \%$ versus $42.0 \%$ ). AF recurrence after successful cardioversion was $36.5 \%$ with dronedarone and $24.3 \%$ with amiodarone. Premature drug discontinuation was less frequent with dronedarone $(10.4 \%$ versus $13.3 \%$ ). The authors concluded that dronedarone was less effective than amiodarone in preventing AF recurrence after direct current cardioversion, but had a better safety profile and a lack of interaction with oral anticoagulants.

A meta-analysis of the aforementioned trials and four placebo-controlled amiodarone trials confirmed the findings of inferior dronedarone efficacy and a better safety profile. ${ }^{40}$

\section{Roles and guidelines}

Based on the evidence, the US Food and Drug Administration (FDA) has licensed dronedarone for use in paroxysmal or persistent AF and atrial flutter with associated cardiovascular risk factors (age $>70$ years, hypertension, diabetes, prior stroke, left atrial diameter $\geq 50 \mathrm{~mm}$, or left ventricular ejection fraction $<40 \%$ ) in patients who are in sinus rhythm or who will be cardioverted. The recommendation is aimed at reducing hospitalizations. Due to the findings of ANDROMEDA, dronedarone is contraindicated in patients with NYHA III or IV heart failure or recent decompensated NYHA II heart failure. Therefore, although dronedarone is intended for patients at high risk of stroke, including those with left ventricular systolic dysfunction, it is contraindicated in those with more than mild heart failure symptoms.

Mostly on the basis of the ATHENA data, the European and US guidelines for AF give dronedarone a class IIa recommendation (level of evidence B) for use in the treatment of nonpermanent AF to reduce hospitalization. Flecainide, propafenone, and sotalol all have restrictions on their use in the context of structural heart disease, so dronedarone has a role in those with structural heart disease but minimal heart failure symptoms (Figure 3). Although amiodarone has greater efficacy than dronedarone in maintaining sinus

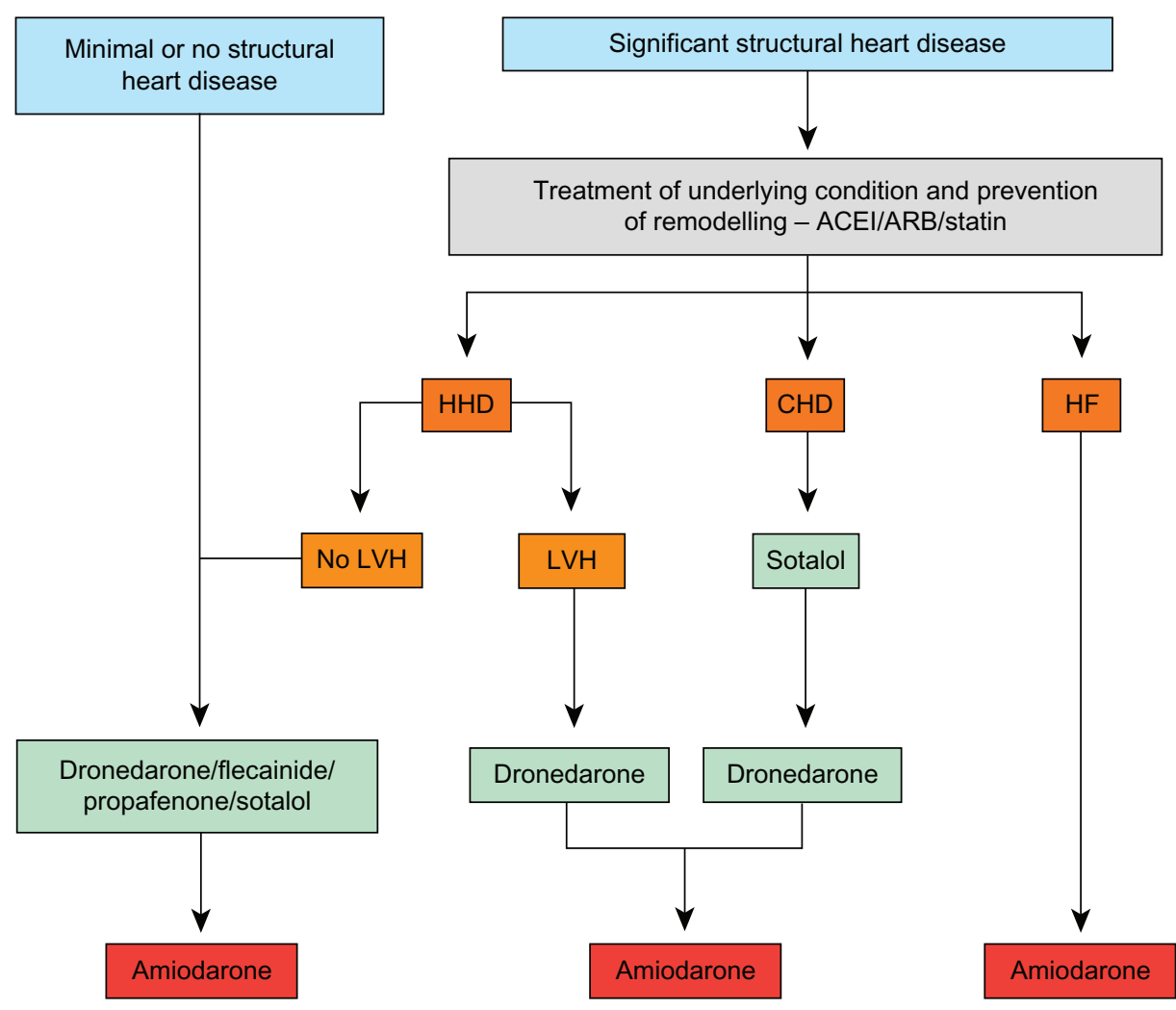

Figure 2 ESC recommendations on choice of antiarrhythmic drug according to underlying pathology.

Abbreviations: ACEl, angiotensin-converting enzyme inhibitor; ARB, angiotensin receptor blocker; HHD, hypertensive heart disease; CHD, coronary heart disease; HF, heart failure; LVH, left ventricular hypertrophy; NYHA, New York Heart Association.

Adapted with permission from Camm A, Lip G, R DC, et al ${ }^{20} .2012$ focused update of the ESC Guidelines for the management of atrial fibrillation. European Heart Journal. 2012;33:2719-47 
Drug

\begin{tabular}{l|l} 
Most effective & $\begin{array}{l}\text { Amiodarone } \\
\text { Dofetilide } \\
\text { Flecainide } \\
\text { Some efficacy }\end{array}$ \\
Propafenone \\
Quinidine \\
Sotalol \\
Dronedarone \\
$\begin{array}{l}\text { No demonstrable } \\
\text { benefit }\end{array}$ \\
Betablocker \\
Verapamil \\
Digoxin
\end{tabular}

OR of recurrence $(95 \% \mathrm{Cl})$

$0.19(0.14-0.27)$

$0.28(0.20-0.38)$

$0.31(0.16-0.60)$

$0.37(0.28-0.48)$

$0.51(0.40-0.65)$

$0.53(0.44-0.65)$

$0.60(0.47-0.76)$

$0.74(0.49-1.13)$

Unable to estimate

Unable to estimate

Figure 3: Agents for the prevention of recurrent AF after DC cardioversion.

Note: **adapted from Lafuente-Lafuente et al ${ }^{23}$ Cochrane Database Syst Rev. 2007 Oct 17;(4):CD005049.

Abberviation: $\mathrm{OR}$, odds ratio; $\mathrm{Cl}$, confidence interval.

rhythm, its toxicity has led to it being reserved for those with AF refractory to other antiarrhythmic drugs.

For patients with NYHA III/IV heart failure, the options for rhythm control are rather limited. Dronedarone is contraindicated, as is flecainide, propafenone, and sotalol. Some physicians are comfortable prescribing these agents for patients who have an intracardiac device in situ (because they are less vulnerable to death resulting from ventricular arrhythmias), although the safety of this remains uncertain. Options remaining for these patients are to accept AF (ie, to attempt rate control, and if this is inadequate to consider atrioventricular node ablation and biventricular pacing), to take long-term amiodarone, or to consider catheter ablation. Accepting AF is a reasonable option, although many patients remain symptomatic despite adequate rate control. Amiodarone has significant side effects associated with long-term use, and for this reason it is becoming less popular for the treatment of atrial arrhythmias. There are limited data available regarding the safety and efficacy of catheter ablation for AF in the context of heart failure. The available data suggest that it is safe and relatively effective in the medium term, and may improve left ventricular function and heart failure symptoms. ${ }^{41-45}$ However, large-scale, randomized, controlled trials examining long-term efficacy are awaited. Therefore, at the moment, catheter ablation of AF in the context of heart failure is only an option for carefully selected patients at centers with adequate expertise.

\section{Safety concerns about dronedarone and fulminant hepatic failure}

The FDA issued a news alert early in 2011 after receiving several case reports of hepatocellular liver injury and hepatic failure in patients treated with dronedarone, including two reports of acute hepatic failure requiring transplantation. ${ }^{46}$ The two cases of acute hepatic failure requiring transplantation occurred at 4.5 and 6 months after initiation of dronedarone in patients with previously normal hepatic serum enzymes. Both patients were female, approximately 70 years of age, and with no etiological cause identified. The explanted livers showed evidence of extensive hepatocellular necrosis. The FDA advised caution and serial monitoring of liver function tests. The European Medicines Agency and the Medicines and Healthcare products Regulatory Agency of the UK have followed the FDA. The FDA has made a general recommendation for serial liver function testing, whereas the Medicines and Healthcare products Regulatory Agency of the UK has given specific advice, ie, testing before use, monthly for the first 6 months, at 9 and 12 months, and annually thereafter. ${ }^{47}$ If alanine transaminase levels are three times the upper limit of normal, they should be repeated after 48-72 hours, and if they remain elevated, the drug should be discontinued.

\section{Summary}

$\mathrm{AF}$ is no longer thought of as a benign condition. From a patient perspective, the priorities for management remain symptom improvement, mortality benefit, stroke 
prevention, and avoidance of medication. Recent evidence suggests early detection and intervention may prevent progression of AF and impact on hard outcomes, such as stroke and death, over the longer term. Given that antiarrhythmic drugs rarely eliminate AF altogether, a reduction in AF burden and therefore symptoms is a reasonable aim. Selection of antiarrhythmic drug is primarily on safety grounds (avoiding proarrhythmia and extracardiac side effects) rather than efficacy. Amiodarone remains the most effective antiarrhythmic drug in maintaining sinus rhythm, although due to its toxicity, many physicians are uncomfortable prescribing it, even in those symptomatic, despite other antiarrhythmic drugs. Amiodarone is now used mostly for limited periods, eg, post cardiac surgery or for very elderly patients.

Dronedarone has better pharmacokinetics, fixed-dosing regimens, outpatient initiation, and an apparently low risk of proarrhythmia, cardiac, and extracardiac toxicity compared with other antiarrhythmic drugs. The European and US guidelines potentially give dronedarone a prominent role in the management of AF. However, many patients may be disappointed by the limited efficacy of dronedarone. Dronedarone is inferior to amiodarone in maintaining sinus rhythm and has efficacy comparable with that of sotalol or flecainide (Figure 3).

Other antiarrhythmic drugs in development include amiodarone analogs (eg, celivarone) and a class III agent called vernakalant. ${ }^{48,49}$ Vernakalant has recently been approved for use in chemical cardioversion for patients with AF of $\leq 7$ days' duration and post-cardiac surgical patients with AF $\leq 3$ days' duration. ${ }^{20}$ Vernakalant preferentially affects atrial ion channels, resulting in increased atrial refractiveness and rate-dependent slowing of atrial contraction, with minimal impact on ventricular repolarization. A head-to-head comparison with intravenous amiodarone showed that vernakalant was markedly superior in restoration of sinus rhythm within 90 minutes $(51.7 \%$ versus $5.2 \% ; P=0.0001)$ and within 4 hours after infusion $(54.4 \%$ versus $22.6 \% ; P=0.0001) .{ }^{50}$ It has a good safety profile immediately and at 24 hours, with most symptoms (taste alteration, sneezing, paraesthesia, and nausea) being transient and resolving within 5-15 minutes. Vernakalant is known to prolong the QTc interval and is contraindicated in those with uncorrected QT $>440 \mathrm{msec}$, severe structural heart disease, severe left ventricular systolic dysfunction (ejection fraction $\leq 35 \%$ ), and an acute coronary syndrome in the last 30 days. The main limitation of vernakalant is that it is only available as an intravenous preparation, and an oral preparation may expand its role to being a "pill-in-the-pocket".
Celivarone (SSR149744C) is a noniodinated benzofuran derivative similar to amiodarone and dronedarone. ${ }^{51,52}$ Two Phase II clinical trials compared celivarone with amiodarone and placebo, ${ }^{53}$ and the results have been disappointing, in that celivarone was not efficacious in the maintenance of sinus rhythm or in cardioverting patients with AF or atrial flutter. Ranolazine, an inhibitor of abnormal late sodium channels, is licensed for use as an antianginal, has a good safety profile, and at present there is no documented occurrence of proarrhythmia. Observational studies suggest this has potential benefit as a "pill-on-the-pocket" for treating paroxysmal AF. ${ }^{54}$

\section{Disclosure}

The authors report no conflicts of interest in this work.

\section{References}

1. Go A, Hylek E, Phillips K, et al. Prevalence of diagnosed atrial fibrillation in adults: national implications for rhythm management and stroke prevention: the AnTicoagulation and Risk Factors in Atrial Fibrillation (ATRIA) study. JAMA. 2001;285:2370-2375.

2. Naccarelli G, Varker H, Lin J, Schulman K. Increasing prevalence of atrial fibrillation and flutter in the United States. Am J Cardiol. 2009; 104:1534-1539.

3. Lip G, Boos C. Antithrombotic treatment in atrial fibrillation. Heart. 2006;92:155-161.

4. Wolf P, Abbott R, Kannel W. Atrial fibrillation as an independent risk factor for stroke: the Framingham Study. Stroke. 1991;22:983-988.

5. Kim MH, Johnston SS, Chu B-C, Dalal MR, Schulman KL. Estimation of total incremental health care costs in patients with atrial fibrillation in the United States. Circ Cardiovasc Qual Outcomes. 2011;4:313-320.

6. Wyse D, Waldo A, DiMarco J, et al. Atrial Fibrillation Follow-up Investigation of Rhythm Management (AFFIRM) Investigators. A comparison of rate control and rhythm control in patients with atrial fibrillation. N Engl J Med. 2002;347:1825-1833.

7. Hagens V, Ranchor A, Van Sonderen E, et al. Effect of rate or rhythm control on quality of life in persistent atrial fibrillation. Results from the Rate Control Versus Electrical Cardioversion (RACE) Study. J Am Coll Cardiol. 2004;43:241-247.

8. Corley S, Epstein A, DiMarco J, et al. Relationships between sinus rhythm, treatment, and survival in the Atrial Fibrillation Follow-Up Investigation of Rhythm Management (AFFIRM) Study. Circulation. 2004;109:1509-1513.

9. Jais P, Cauchemez B, Macle L, et al. Catheter ablation versus antiarrhythmic drugs for atrial fibrillation: the A4 study. Circulation. 2008;118:2498-2505.

10. Oral H, Pappone C, Chugh A, et al. Circumferential pulmonary-vein ablation for chronic atrial fibrillation. $N$ Engl J Med. 2006;453: 934-941.

11. Pappone C, Augello G, Sala S, et al. A randomized trial of circumferential pulmonary vein ablation versus antiarrhythmic drug therapy in paroxysmal atrial fibrillation: the APAF Study. J Am Coll Cardiol. 2006;48:2340-2347.

12. Stabile G, Bertaglia E, Senatore G, et al. Catheter ablation treatment in patients with drug-refractory atrial fibrillation: a prospective, multicentre, randomized, controlled study (Catheter Ablation For The Cure Of Atrial Fibrillation Study). Eur Heart J. 2006;27:216-221.

13. Wazni O, Marrouche N, Martin D, et al. Radiofrequency ablation vs antiarrhythmic drugs as first-line treatment of symptomatic atrial fibrillation: a randomized trial. JAMA. 2005;293:2634-2640. 
14. Wilber D, Pappone C, Neuzil P, et al. Comparison of antiarrhythmic drug therapy and radiofrequency catheter ablation in patients with paroxysmal atrial fibrillation: a randomized controlled trial. JAMA. 2010;303:333-340.

15. Bunch T, Crandall B, Weiss J, et al. Patients treated with catheter ablation for atrial fibrillation have long-term rates of death, stroke, and dementia similar to patients without atrial fibrillation. $J$ Cardiovasc Electrophysiol. 2011;22:839-845.

16. Hunter RJ, McCready J, Diab I, et al. Maintenance of sinus rhythm with an ablation strategy in patients with atrial fibrillation is associated with a lower risk of stroke and death. Heart. 2012;98:48-53.

17. Pappone C, Radinovic A, Manguso F, et al. Atrial fibrillation progression and management: a 5-year prospective follow-up study. Heart Rhythm. 2008;5:1501-1507.

18. Pappone C, Rosanio S, Augello G, et al. Mortality, morbidity, and quality of life after circumferential pulmonary vein ablation for atrial fibrillation: outcomes from a controlled nonrandomized long-term study. J Am Coll Cardiol. 2003;42:185-197.

19. Themistoclakis S, Corrado A, Marchlinski F, et al. The risk of thromboembolism and need for oral anticoagulation after successful atrial fibrillation ablation. J Am Coll Cardiol. 2010;55:735-743.

20. Camm A, Lip GY, De Caterina R, et al. 2012 focused update of the ESC guidelines for the management of atrial fibrillation. Eur Heart J. 2012;33:2719-2747.

21. Calkins H, Brugada J, Packer D, et al. HRS/EHRA/ECAS expert consensus statement on catheter and surgical ablation of atrial fibrillation: recommendations for personnel, policy, procedures and follow-up. A report of the Heart Rhythm Society (HRS) Task Force on Catheter and Surgical Ablation of Atrial Fibrillation developed in partnership with the European Heart Rhythm Association (EHRA) and the European Cardiac Arrhythmia Society (ECAS); in collaboration with the American College of Cardiology (ACC), American Heart Association (AHA), and the Society of Thoracic Surgeons (STS). Endorsed and approved by the governing bodies of the American College of Cardiology, the American Heart Association, the European Cardiac Arrhythmia Society, the European Heart Rhythm Association, the Society of Thoracic Surgeons, and the Heart Rhythm Society. Europace. 2007;9:335-379.

22. Hunter R, Berriman T, Diab I, et al. Long term efficacy of catheter ablation for AF: impact of additional targeting of fractionated electrograms. Heart. 2010;96:1372-1378.

23. Lafuente-Lafuente C, Mouly S, Longas-Tejero M, et al. Antiarrhythmics for maintaining sinus rhythm after cardioversion of atrial fibrillation. Cochrane Database Syst Rev. 2007;5:CD005049.

24. Wann L, Curtis A, January C, et al. ACCF/AHA/HRS focused update on the management of patients with atrial fibrillation (Updating the 2006 Guideline): a report of the American College of Cardiology Foundation/ American Heart Association Task Force on practice guidelines. J Am Coll Cardiol. 2011;57:223-242.

25. Armstrong R, Waters E, Jackson N, et al. Guidelines for Systematic Reviews of Health Promotion and Public Health Interventions. Version 2. Melbourne, Australia: Melbourne University, Australia; 2007.

26. Kathofer S, Thomas D, Karle C. The novel antiarrhythmic drug dronedarone: comparison with amiodarone. Cardiovasc Drug Rev. 2005;23:217-230.

27. Wegener F, Ehrlich J, Hohnloser S. Dronedarone: an emerging agent with rhythm- and rate-controlling effects. J Cardiovasc Electrophysiol. 2006;17:E11.

28. Gautier P, Guillemare E, Marion A, Bertrand J-P, Tourneur Y, Nisato D. Electrophysiologic characterization of dronedarone in guinea pig ventricular cells. J Cardiovasc Pharmacol. 2003;41:191-202.

29. Hodeige D, Heyndrickx J, Chatelain P, Manning A. ST 33589, a new amiodarone-like anti-arrhythmic agent: anti-adrenoceptor activity in anaesthetized and conscious dogs. Eur J Pharmacol. 1995;279: 25-32.

30. Hohnloser S, Crijns H, van Eickels M, et al. Effect of dronedarone on cardiovascular events in atrial fibrillation. N Engl J Med. 2009;360: $668-678$.
31. Oyetayo O, Rogers C, Hofmann P. Dronedarone: a new antiarrhythmic agent. Pharmacotherapy. 2010;30:904-915.

32. Touboul P, Brugada J, Capucci A, Crijns H, Edvardsson N, Hohnloser S. Dronedarone for prevention of atrial fibrillation: a dose-ranging study. Eur Heart J. 2003;24:1481-1487.

33. Singh B, Connolly S, Crijns H, et al. Dronedarone for maintenance of sinus rhythm in atrial fibrillation or flutter. $N$ Engl $J$ Med. 2007;357:987-999.

34. Camm A, Reiffel J. Defining endpoints in clinical trials on atrial fibrillation. Eur Heart J. 2008;10 Suppl H:H55-H78.

35. Davy J, Herold M, Hoglund C, et al. ERATO Study Investigators. Dronedarone for the control of ventricular rate in permanent atrial fibrillation: the efficacy and safety of Dronedarone for the control of ventricular rate during atrial fibrillation (ERATO) study. Am Heart $J$. 2008; 156:527. e1-e9.

36. Kober L, Torp-Pedersen C, McMurray J, et al. Increased mortality after dronedarone therapy for severe heart failure. $N$ Engl $J$ Med. 2008;358:2678-2687.

37. Tschuppert Y, Buclin T, Rothuizen L, et al. Effect of dronedarone on renal function in healthy subjects. $\mathrm{Br} J$ Clin Pharmacol. 2007;64:785-791.

38. Connolly SJ, Camm AJ, Halperin JL, et al. Dronedarone in high-risk permanent atrial fibrillation. N Engl J Med. 2011;365:2268-2276.

39. Le Heuzey J, De Ferrari G, Radzik D, Santini M, Zhu J, Davy J. A short-term, randomized, double-blind, parallel-group study to evaluate the efficacy and safety of dronedarone versus amiodarone in patients with persistent atrial fibrillation: the DIONYSOS study. J Cardiovasc Electrophysiol. 2010;21:597-605.

40. Piccini J, Hasselblad V, Peterson E, et al. Comparative efficacy of dronedarone and amiodarone for the maintenance of sinus rhythm in patients with atrial fibrillation. J Am Coll Cardiol. 2009;54:1089-1095.

41. Gentlesk P, Sauer W, Gerstenfeld E, et al. Reversal of left ventricular dysfunction following ablation of atrial fibrillation. $J$ Cardiovasc Electrophysiol. 2007;18:9-14.

42. Hsu L, Jais P, Sanders P, et al. Catheter ablation for atrial fibrillation in congestive heart failure. $N$ Engl J Med. 2004;351:2373-2383.

43. Khan M, Jais P, Cummings J, et al. Pulmonary-vein isolation for atrial fibrillation in patients with heart failure. $N$ Engl J Med. 2008;359: $1778-1785$.

44. Nademanee K, Schwab M, Kosar E, et al. Clinical outcomes of catheter substrate ablation for high-risk patients with atrial fibrillation. J Am Coll Cardiol. 2008;51:843-849.

45. Tondo C, Mantica M, Russo G, et al. Pulmonary vein vestibule ablation for the control of atrial fibrillation in patients with impaired left ventricular function. Pacing Clin Electrophysiol. 2006;29: 962-970

46. FDA Drug Safety Communication: Severe liver injury associated with the use of dronedarone (marketed as Multaq). 2011. Available from: http://www.fda.gov/Drugs/DrugSafety/ucm240011.htm. Accessed November 13, 2012.

47. Medicines and Healthcare products Regulatory Age. Risk of adverse liver reactions - advice for healthcare professionals. 2011. Available from: http://www.mhra.gov.uk/Safetyinformation/DrugSafetyUpdate/ CON108677. Accessed November 13, 2012.

48. Torp-Pedersen C, Raev D, Dickinson G, Butterfield N, Mangal B, Beatch G. A randomized placebo-controlled study of vernakalant (oral) for the prevention of atrial fibrillation recurrence post-cardioversion. Circ Arrhythm Electrophysiol. 2011;4:637-643.

49. Verma A. Alternatives to amiodarone: search for the holy grail. Europace. 2009;11:402-404.

50. Camm A, Capucci A, Hohnloser S, et al. AVRO Investigators. A randomized active-controlled study comparing the efficacy and safety of vernakalant to amiodarone in recent-onset atrial fibrillation. $J \mathrm{Am}$ Coll Cardiol. 2011;57:313-321.

51. Cosnier-Pucheu S, Roccon A, Rizzoli G, et al. SSR149744, a new antiarrhythmic drug, prevents experimental induced atrial fibrillation. Eur Heart J. 2003;2:53-54. 
52. Kowey P, Aliot E, Capucci A, et al. Placebo-controlled double-blind dose-ranging study of the efficacy and safety of SSR149744c in patients with recent atrial fibrillation/flutter. Heart Rhythm. 2007;4:S72.

53. Khitri A, Aliot E, Capucci A, et al. Celivarone for maintenance of sinus rhythm and conversion of atrial fibrillation/flutter. J Cardiovasc Electrophysiol. 2012;23:462-472.
54. Murdock D, Kersten M, Kaliebe J, Larrain G. The use of oral ranolazine to convert new or paroxysmal atrial fibrillation: a review of experience with implications for possible "pill in the pocket" approach to atrial fibrillation. Indian Pacing Electrophysiol J. 2009 Sep-Oct; 9(5): 260-267. PMCID: PMC2735158.

\section{Publish your work in this journal}

Patient Related Outcome Measures is an international, peer-reviewed, open access journal focusing on treatment outcomes specifically relevant to patients. All aspects of patient care are addressed within the journal and practitioners from all disciplines are invited to submit their work as well as healthcare researchers and patient support groups.
The manuscript management system is completely online and includes a very quick and fair peer-review system. Visit http://www.dovepress. com/testimonials.php to read real quotes from published authors.

Submit your manuscript here: http://www.dovepress.com/patient-related-outcome-measures-journal 